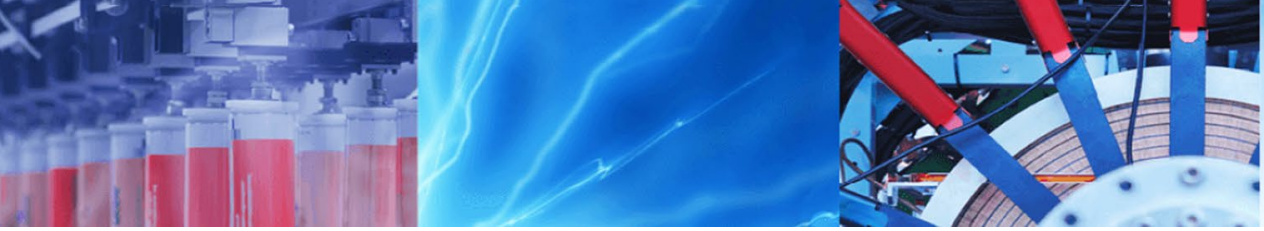

Research Article

\title{
Study of the biosynthesis of gold nanoparticles by using several saprophytic fungi
}

\author{
Samaneh Iranmanesh ${ }^{1}$ (I) $\cdot$ Gholam Hosein Shahidi Bonjar ${ }^{2} \cdot$ Amin Baghizadeh $^{1}$
}

Received: 15 July 2020 / Accepted: 14 October 2020 / Published online: 21 October 2020

(c) Springer Nature Switzerland AG 2020

\begin{abstract}
Gold nanoparticles have wide applications, such as cancer diagnosis and treatment, drug transfer, gene transfer, and DNA and protein detection. Fungi are good options for the biosynthesis of nanoparticles, which is due to their ability to secrete a large quantity of enzymes. The purpose of the present study is to investigate the ability of several saprophytic fungi in the production of gold nanoparticles. The biomass of each fungus was first mixed with a $10^{-3}$ molar $\mathrm{HAuCL}_{4}$ solution and was subsequently heated for $24 \mathrm{~h}$. The Change in the color of the reaction solutions from yellow to violet was symptomatic of the synthesis of gold nanoparticles. In cases where the above assessment (i.e., color change) was positive, the synthesis of gold nanoparticles was further investigated by using spectrophotometry UV-vis, X-ray diffraction and TEM electron microscope. The observation of the maximum absorption peak at a wavelength of about $540 \mathrm{~nm}$ in the UV-vis spectrum confirmed the production of gold nanoparticles. Moreover, the XRD analysis of the nanoparticles proved that the nanoparticles synthesized are gold nanocrystals. The TEM electron microscope technique was used to determine the shape and size distribution of the nanoparticles. Based on electromicrographs, the nanoparticles were spherical, triangular and polygonal.
\end{abstract}

Keywords Fungi · Gold nanoparticles · Synthesis · TEM · XRD

\section{Introduction}

Nanotechnology is one of the growing branches of science and involves the production, manipulation and the use of materials at a scale smaller than microns. The properties of materials in nano and micro scales are not identical. The synthesis of nanomaterials is important due to their unusual optical, chemical and electrical properties. Gold nanoparticles are widely used in medical fields such as cancer diagnosis and treatment, drug treatment, biosensors, DNA and protein detection, and the removal of pathogenic microorganisms from contaminated water. Although nanoscale materials are mainly produced by chemical industries, living organisms are an important source of these materials. This is because chemical methods are time-consuming and costly. Nanoparticles can be produced by bacteria, actinomycetes, fungi, and plants. Throughout the world, numerous studies have been conducted which employ microorganisms such as bacteria and actinomycetes (prokaryotes), as well as algae, yeasts, fungi, and organic plants (eukaryotes) for the synthesis of metal nanoparticles. Due to Fungi's simple and inexpensive cultivation in both industrial and laboratory scales, their release of enzymes, safety for the environment, easy manipulation, the ability to resuscitate one or more metal ions, the production of high volumes of nanoparticles and relatively lower cost, they are usually preferred to other microorganisms.

$\checkmark$ Samaneh Iranmanesh, sp.iranmanesh@gmail.com | ${ }^{1}$ Department of Biotechnology, Institute of Science and High Technology and Environmental Sciences, Graduate University of Advanced Technology, Kerman, Iran. ${ }^{2}$ Department of Plant Pathology, College of Agriculture, Shahid Bahonar University of Kerman, Kerman, Iran. 
In recent studies, fungi such as Fusarium, Verticillium, and Aspergillus have been used to synthesize metal nanoparticles such as copper, cadmium, silver, and gold [1]. In the vicinity of gold ions, fungi produce large quantities of nanoparticles. Mukherjee et al. [2] employed the fungus Verticillium sp. to synthesize gold nanoparticles. The maximum absorption peak in the UV-vis diagram was $540 \mathrm{~nm}$. In addition, they reported average size of $20 \mathrm{~nm}$ for nanoparticles. They also used the fungus Fusarium oxysporum for extracellular synthesis of gold nanoparticles [3]. The UV-vis spectrometry showed the maximum absorption peak at $545 \mathrm{~nm}$. Additionally, they reported that the synthesized nanoparticles were spherical or triangular and fall within the range of $8-40 \mathrm{~nm}$. By using the endophytic fungus Colletotrichum which was isolated from the geranium leaves, Shankar et al. [4] produced gold nanoparticles. Ahmad et al. [5] also employed the fungus Trichothecium to produce gold nanoparticles. They reported that by controlling the reaction conditions, the synthesis of gold nanoparticles can be intracellular or extracellular. Bhambure et al. [6] employed the fungus Aspergillus niger for extracellular biosynthesis of gold nanoparticles. The average size of the produced nanoparticles was $12.79 \pm 5.61 \mathrm{~nm}$. They found that the synthesized nanoparticles are very stable in the solution. Philip [7] produced $\mathrm{Au}, \mathrm{Ag}$ and $\mathrm{Au}-\mathrm{Ag}$ nanoparticles by employing the extract of Volvariella volvacea. The shape of the nanoparticles was triangular, spherical and hexagonal. Moreover, their dimensions were in the range of 20-150 $\mathrm{nm}$ and the morphology of the nanoparticles was dependent on the extract temperature. At the lower temperature, the nanoparticles were almost hexagonal while at the higher temperature dendrite/ fractal structures were observed. Binupriya et al. [8] used the fungus Rhizopus stolonifer for biosynthesis of gold and silver nanoparticles. The formation of nanoparticles was confirmed by UV-vis spectrum, TEM and XRD analyses. The gold nanoparticles were in the range of $25-30 \mathrm{~nm}$. Soni and Prakash [9] used the fungus Aspergillus niger to synthesize gold nanoparticles. The UV-vis spectrometry showed the maximum absorption peak at $530 \mathrm{~nm}$. In addition, the size of the nanoparticles was in the range of $10-30 \mathrm{~nm}$. Sarkar et al. [10] produced gold nanoparticles by using the fungus Alternaria alternata. By employing the Fourier transform infrared spectroscopy, the existence of a protein shell outside the nanoparticles was confirmed which had a supporting role for their stability. By using the fungus Fusarium oxysporum, Thakker et al. [11] performed a fast extracellular synthesis of gold nanoparticles. The average size of the nanoparticles was $22 \mathrm{~nm}$, and these nanoparticles exhibited antimicrobial activity against Pseudomonas sp.. Gopinath and Arumugam [12] reported that the fungus Fusarium solani can be used for the fast extracellular synthesis of gold nanoparticles. The size of the nanoparticles ranged from 20-50 nm. Joshi et al. [13] employed the marine endophytic fungus Cladosporium cladosporioides for the synthesis of gold nanoparticles with a mean size of $100 \mathrm{~nm}$ and reported strong antioxidant and antibacterial properties for them. Patil and Kim [14], utilizing marine microorganisms such as bacteria, fungi, and microalgae, produced nanoparticles. For instance, the fungi Aspergillus sydowii and Aspergillus terreus had the capability of producing gold nanoparticles. Pourali et al. [15] considered the biosynthesis of gold nanoparticles by Fusarium oxysporum and focused on the possibility of nanoparticles conjugation with $\beta$-Lactam antibiotics. They reported high tendency of conjugation with antibiotics for nanoparticles. Sreedharan et al. [16] employed the fungus Macrophomina phaseolina for synthesis of gold nanoparticles. The formation of nanoparticles was confirmed by UV-vis spectrum, TEM and EDX analyses. The sizes of the nanoparticles were in the range $14-16 \mathrm{~nm}$.

Based on the literature review, many studies have been carried out into the synthesis of gold nanoparticles by employing fungi. However, the ability of some fungi in the synthesis of gold nanoparticles still remains unknown. In this study, the ability of 17 fungi in the synthesis of gold nanoparticles is evaluated. In the first stage, the production of gold nanoparticles is distinguished by the color change of the mixture of biomass and chlorine solution. Subsequently, out of the fungi which are able to produce nanoparticles, 5 fungi are selected for further analysis. The analyses include UV-vis spectrometry, transient electron microscope (TEM) and X-ray diffraction. In the present study, in one case (Verticillium dahliae), the species of the selected fungus is different from those in the previous studies. In addition, to the best knowledge of the authors, the fungus Rhizoctonia solani has not been employed for the synthesis of gold nanoparticles in the published literature.

\section{Materials and Methods}

Tables 1 and 2 represent the material and the fungi used in this study, respectively. The fungi were first cultured on a Potato Dextrose Agar (PDA) medium. Subsequently, they were incubated at $27^{\circ} \mathrm{C}$ for 3 to 7 days. These cultures were used to produce fungal biomasses in a Glucose Yeast Peptone (GYP) liquid culture medium using a rotary shaker with rpm of 120 for a period of 5-7 days. After this period which resulted in well fungal growth, the biomasses were collected and well rinsed with distilled water by using Whatman filter paper No. 1 and a glass funnel to exclude the remains of

Table 1 The list of the used materials

\begin{tabular}{ll}
\hline No. & Material \\
\hline 1 & HAuCL $_{4}$ solution \\
2 & PDA culture medium $^{2}$ \\
3 & GYP culture medium \\
\hline
\end{tabular}


Table 2 The list of the studied fungi

\begin{tabular}{ll}
\hline No & Fungus \\
\hline 1 & Fusarium oxysporum \\
2 & Sclerotinia sclerotium \\
3 & Aspergillus flavus \\
4 & Paecilomyces variotii \\
5 & Colletotrichum gloeosporioides \\
6 & Rhizoctonia solani \\
7 & Verticilium dahliae \\
8 & Botrytis oryzae \\
9 & Stemphilium sp. \\
10 & Peceaiomyces sp. \\
11 & Gaeumanomyces sp. \\
12 & Metarhizium $\mathrm{sp}$. \\
\hline
\end{tabular}

the culture medium. To ensure the exclusion of secondary metabolites and culture media, the samples then subjected to three cycles of centrifugation. Each cycle runs for $10 \mathrm{~min}$ at $10,000 \mathrm{rpm}$. At the end of each cycle, the supernatant discarded and the pellet suspended in distilled water. Suspension of biomasses in water was used for the evaluation of their bioactivity in the production of gold nanoparticles. To this end, four samples were prepared as follows. To the first tube (control), for $0.5 \mathrm{~g}$ of the wet biomass, $2 \mathrm{ml}$ distilled water was added. To the second tube, for half a gram of the dead fungal biomass (the fungus biomass is killed by the autoclave), $2 \mathrm{ml} \mathrm{HAuCL}_{4}$ solution with a concentration of $10^{-3}$ molar was added. To the third tube, for half a gram of the wet biomass, $2 \mathrm{ml} \mathrm{HAuCL}_{4}$ solution with a concentration of $10^{-3}$ molar was added. Finally, to the fourth tube for $1 \mathrm{ml}$ of the culture medium (after passing from the filter paper), $2 \mathrm{ml}$ of $\mathrm{HAuCl}_{4}$ solution with a concentration of $10^{-3}$ molar was added. These tubes were placed inside a rotary shaker with a revolution of $120 \mathrm{rpm}$ for $24-72 \mathrm{~h}$ at a temperature of $27^{\circ} \mathrm{C}$.

\section{Results and Discussion}

\subsection{Initial evaluation of the synthesis of gold nanoparticles}

The simplest method for the purpose of the evaluation of the synthesis of gold nanoparticles is the observational method. The synthesis of gold nanoparticles is recognized by the color change of the mixture of biomass and $\mathrm{HAuCl}_{4}$ from yellow to violet. This color change is due to the vibrational excitation of the surface plasmon by the nanoparticles, which is within the visible wavelength range and near $540 \mathrm{~nm}$ [17]. In the current study, 12 fungi (as listed in Table 2) were evaluated. The color change was observed for 8 fungi. Based on this evaluation, it was concluded that only the fungi Stemphilium, Metarhizium, Gaeumanomyces, and Botrytisoryzae are not capable of synthesizing gold nanoparticles. The evaluation results for four selected fungi have been presented in Fig. 1. In each frame, from left to right, the tubes contain the live fungus biomass in the distilled water (control tube), the dead fungus biomass incubated by $\mathrm{HAuCl}_{4}$, the live fungus biomass incubated by $\mathrm{HAuCl}_{4}$ and the PDA medium incubated by $\mathrm{HAuCl}_{4}$. In comparison with the control tube, the change in the color of colonies after $24 \mathrm{~h}$ at a temperature of $29^{\circ} \mathrm{C}$ can be observed in Fig. 1.

\subsection{UV-Vis Spectrometry}

The UV-vis spectrometry is used to confirm the production of gold nanoparticles. In this analysis, the absorption of the ultraviolet ray at different wavelengths is considered. Since gold has an absorption spectrum in the range of $500-600 \mathrm{~nm}$, the UV-vis spectrometry is considered for wavelengths in the range of $400-700 \mathrm{~nm}$. For this purpose, about $1 \mathrm{ml}$ of the supernatant of the reaction solution was isolated. Afterwards, its absorption spectrum was measured for the wavelengths in the range of 400-700 nm. The important evidence suggesting the formation of gold nanoparticles is the maximum absorption peak at a wavelength of about $540 \mathrm{~nm}$ [2]. Figures 2 and 3 show the results of the UV-vis spectrometry for the 8 fungi which passed the previous evaluation successfully. All of the graphs have a maximum absorption peak near the $540 \mathrm{~nm}$ wavelength.

Table 3 shows the wavelength of the maximum absorption for the examined fungi. The maximum and minimum amounts of absorption belonged to the fungus Rhizoctonia solani and Peceaiomyces sp., respectively.

\subsection{Transient Electron Microscope (TEM) Analysis}

Out of the 8 fungi which were able to produce gold nanoparticles, 4 fungi were selected for further analysis by the transient electron microscope (TEM). By using the TEM micrographs, it can be seen that the synthesis of the nanoparticles is intracellular or extracellular. Moreover, the shape and the size distribution of the nanoparticles are determined. Figure 4 presents the TEM micrographs for gold nanoparticles synthesized by the fungi Fusarium oxysporum, Aspergillus flavus, Rhizoctonia solani, and Verticillium dahliae. According to the TEM micrographs, the synthesized nanoparticles are intercellular.

The nanoparticles with different sizes and shapes can be observed in these figures. Based on these micrographs, the nanoparticles produced by fungi Aspergillus flavus, Rhizoctonia solani are mainly spherical whereas the 

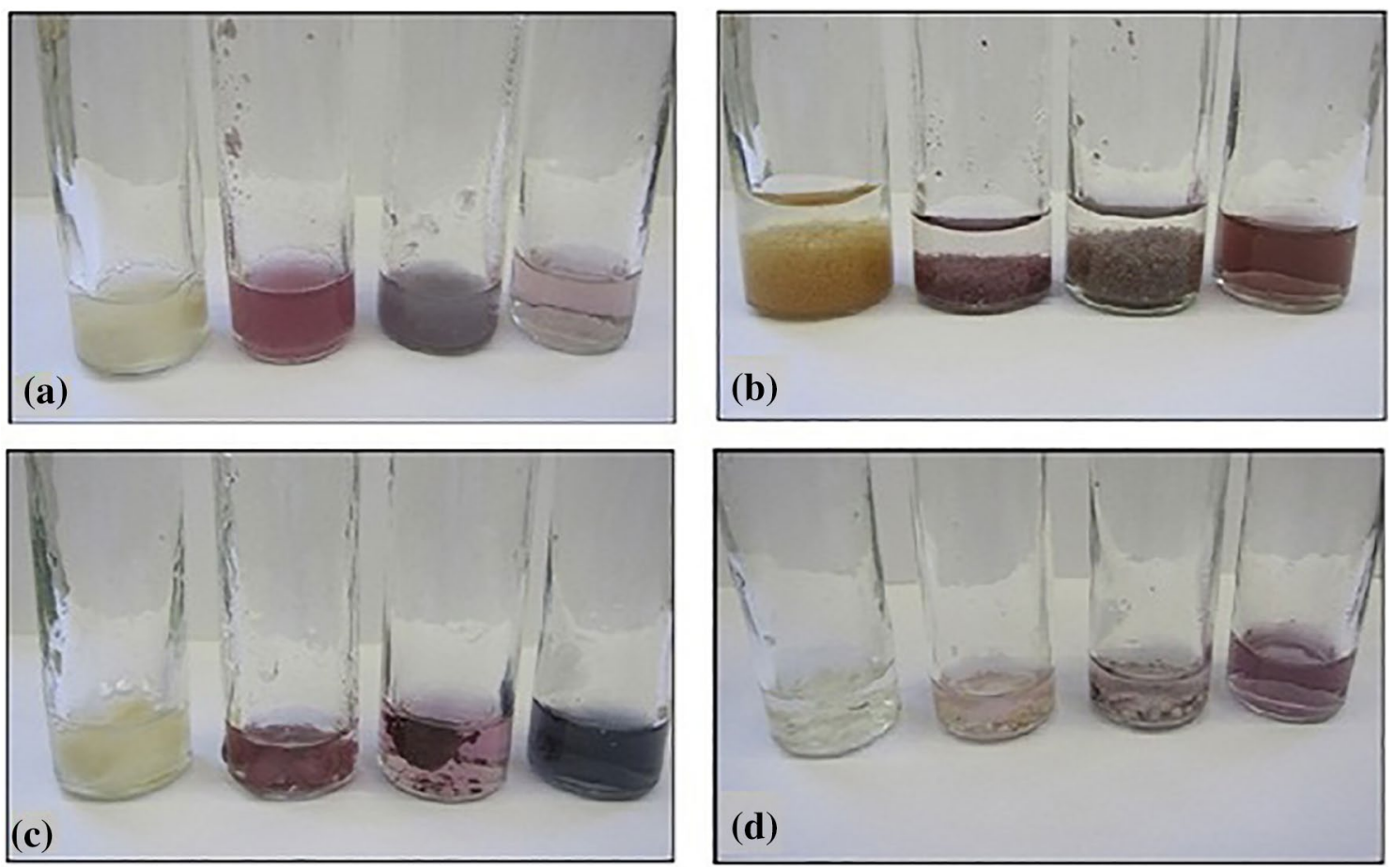

Fig. 1 The production of gold nanoparticles by the fungi a Fusarium oxysporum, b Aspergillus flavus c Rhizoctonia solani, d Verticillium dahliae

nanoparticles produced by Fusarium oxysporum are triangular and spherical and those produced by Verticillium dahliae are often polygonal. The TEM micrographs are used to determine the size distribution of gold nanoparticles. The results appear in Fig. 5 for the examined fungi. It can be observed that the maximum number of the synthesized nanoparticles belongs to the fungus Rhizoctonia solani. The mean size of nanoparticles for the fungi Aspergillus flavus, and Rhizoctonia solani is about $30 \mathrm{~nm}$ and for the fungi Fusarium oxysporum and Verticillium dahliae, it is 20 and $40 \mathrm{~nm}$, respectively. Therefore, the smallest and largest sizes of the nanoparticles belong to the fungi Fusarium oxysporum and Verticillium dahliae, respectively. As it is observed, different fungi produce nanoparticles with different sizes and shapes. These differences are probably related to the kind and amount of secreted enzymes by different fungi. However, more researches are required to understand the mechanism of this phenomenon.

\subsection{X-ray diffraction (XRD) Analysis}

X-ray diffraction (XRD) is a direct method for the determination of the phase-type and crystalline structure of materials. To prepare for XRD analysis, the samples (after gold nanoparticle synthesis) are centrifuged with a revolution of $18,000 \mathrm{rpm}$ for $20 \mathrm{~min}$. Subsequently, the supernatant is discarded and the deposited nanoparticles are sent for the
XRD analysis. Figures 6 and 7 show the XRD analysis results for the examined fungi. The diagrams represent the intensity of the diffracted beam versus the diffraction angle. In all the diagrams, the peaks are observed at the diffraction angles $38.09^{\circ}, 44.3^{\circ}, 64.45^{\circ}$ and $77.37^{\circ}$, which correspond to the crystalline plates (111), (200), (220), and (311), respectively. This analysis proves that the synthesized particles are gold nanocrystals [9]. In all the samples, before the diffraction angle $38.09^{\circ}$, some peaks are observed. The reason is that in addition to gold nanoparticles, the samples contain organic materials.

\subsection{Verification of the Results}

In this section, for the purpose of verification, the results of the present work are related to the relevant findings in the literature. Mukherjee et al. [3] synthesized gold nanoparticles by the fungus Fusarium oxysporum. They used the $\mathrm{HAuCl}_{4}$ solution with a concentration of $10^{-3} \mathrm{M}$. The UV-vis spectrometry showed the maximum absorption peak at $545 \mathrm{~nm}$. Moreover, they reported that the synthesized nanoparticles were spherical or triangular and in the range of 8-40 $\mathrm{nm}$. In the present study, the maximum absorption peak was $530 \mathrm{~nm}$. In addition, the nanoparticles were synthesized in the range of 5-41 nm, with spherical or triangular shapes. These agreements confirm the validity of our results.

\section{SN Applied Sciences}




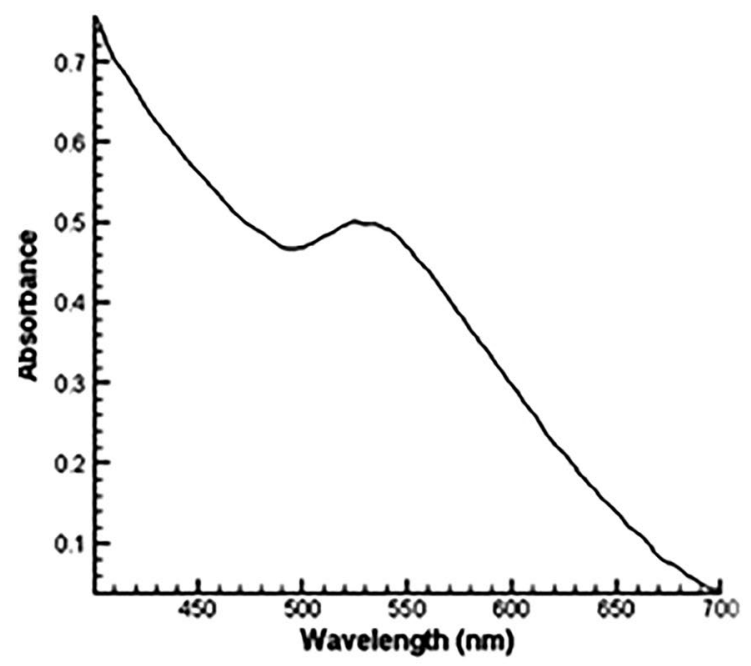

(a)

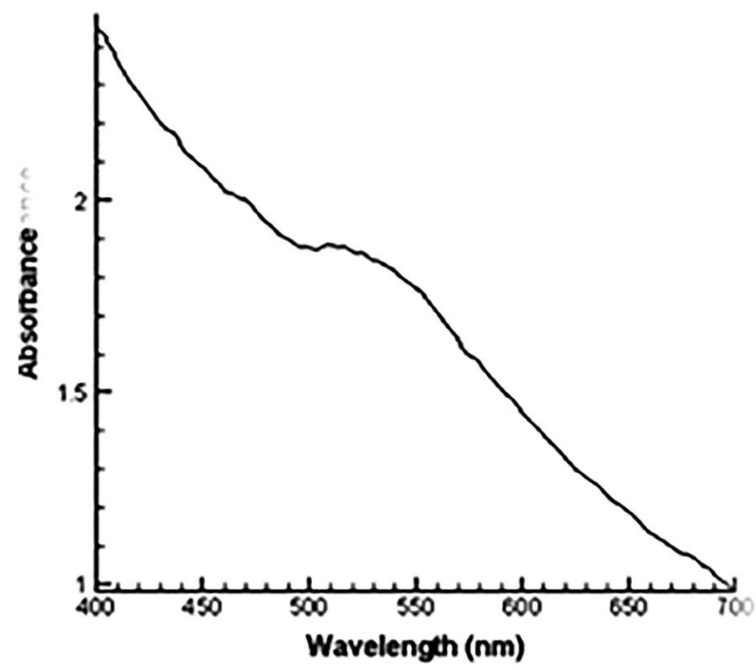

(c)

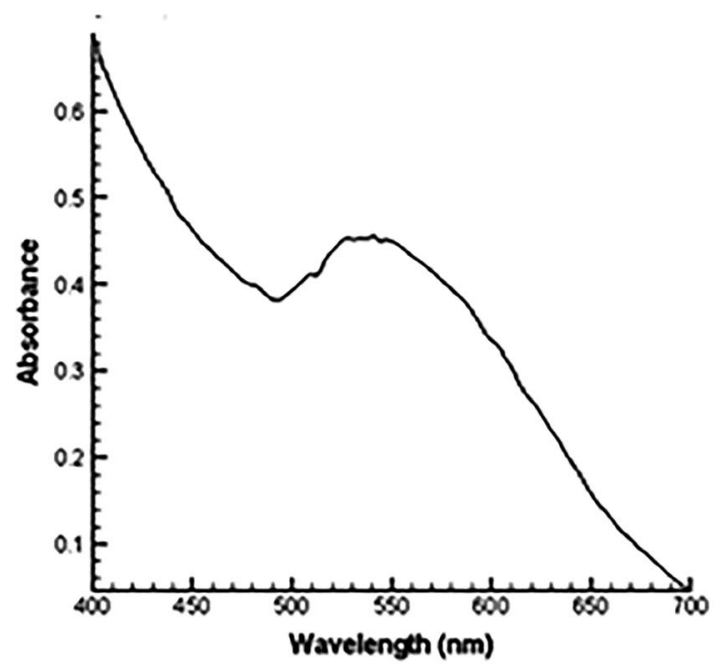

(b)

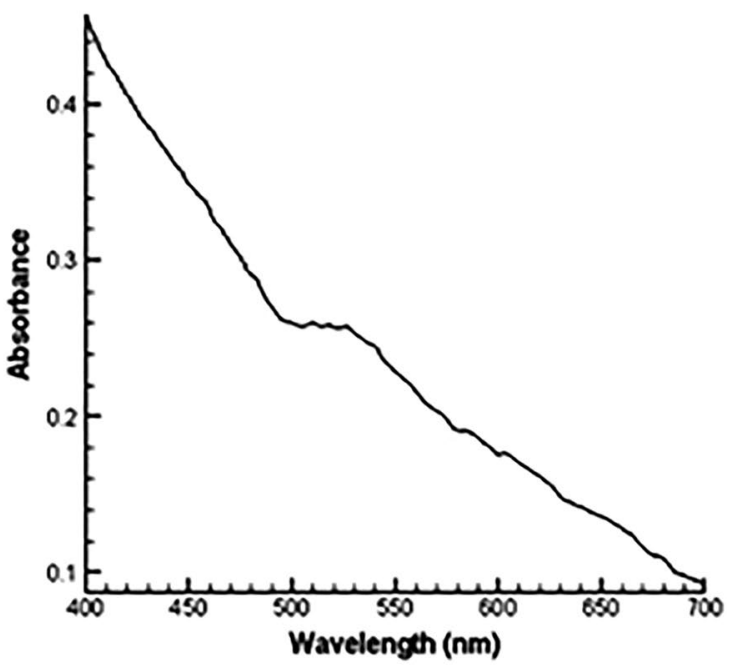

(d)

Fig. 2 The UV-vis spectrometry analysis for the fungi a Fusarium oxysporum, b Aspergillus flavus, c Rhizoctonia solani, d Colletotrichum gloeosporioides

Soni and Prakash [9] used the fungus Aspergillus niger to synthesize gold nanoparticles. The concentration of the $\mathrm{HAuCl}_{4}$ solution was $10^{-3} \mathrm{M}$. The UV-vis spectrometry showed the maximum absorption peak at $530 \mathrm{~nm}$. Besides, the size of the nanoparticles was in the range of 10-30 $\mathrm{nm}$. In the present study, the maximum absorption peak for Aspergillus niger was 537. The fungus Aspergillus flavus was also examined in the current study. The maximum absorption peak was observed at $534 \mathrm{~nm}$, and the nanoparticles were in the range of $5-51 \mathrm{~nm}$. It could be seen that Aspergillus flavus produces larger nanoparticles, as compared with Aspergillus niger. Therefore, it is concluded that the species of the fungus can affect the size of the synthesized nanoparticles.
Mukherjee et al. [2] employed the fungus Verticillium sp. to synthesize gold nanoparticles. In their experiment, the concentration of the $\mathrm{HAuCl}_{4}$ solution was $10^{-4} \mathrm{M}$. The maximum absorption peak in the UV-vis diagram was $540 \mathrm{~nm}$. They also reported average size of $20 \mathrm{~nm}$ for the nanoparticles. In the study undertaken here, the experiments were carried out on the fungus Verticillium dahliae. The maximum absorption peak and the average size of the nanoparticles were 534 and $40 \mathrm{~nm}$, respectively. In the present study, the average size of the nanoparticles is larger. This is probably due to the difference in the concentration of the $\mathrm{HAuCl}_{4}$ solution used in the two studies. The use of a solution with higher molarity results in larger nanoparticles. Besides, Mukherjee et al. did not specify the species 


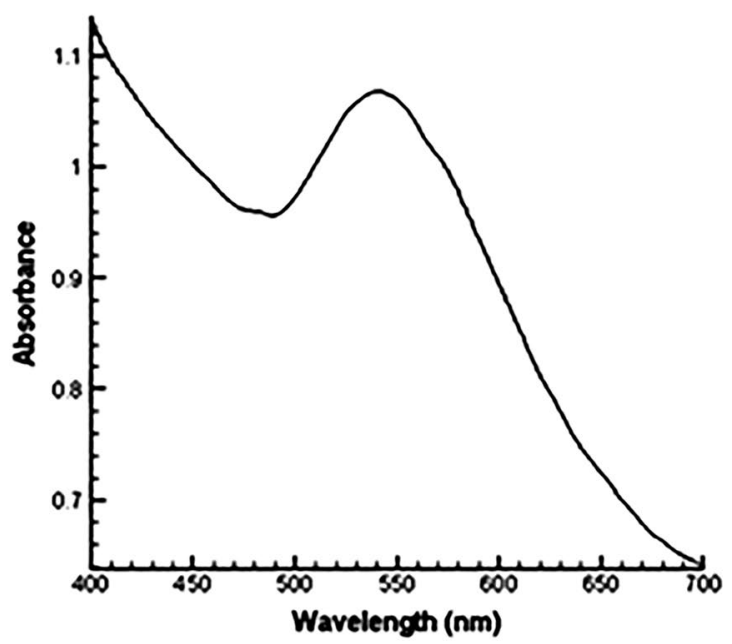

(a)

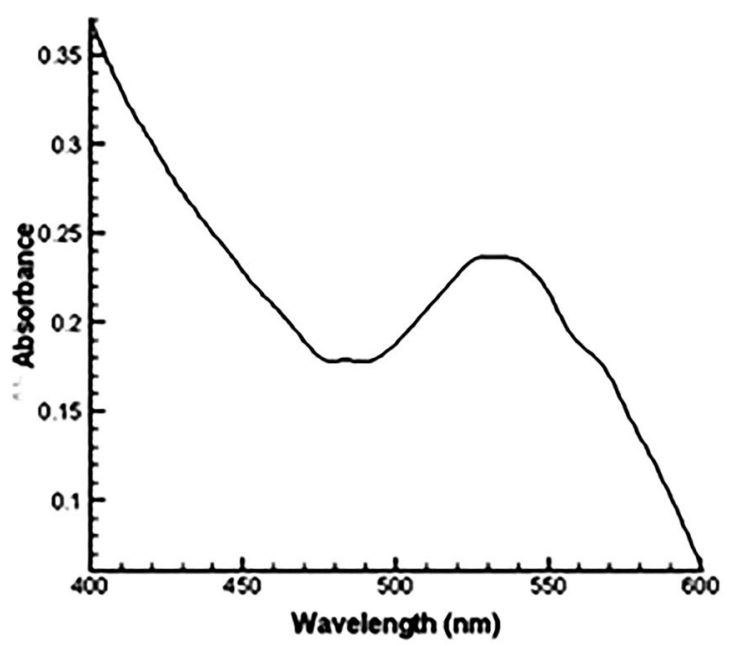

(c)

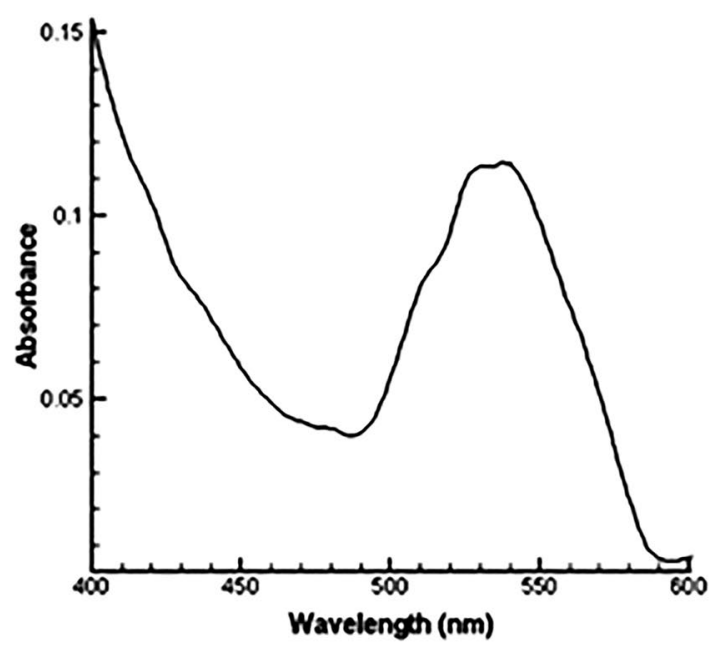

(b)

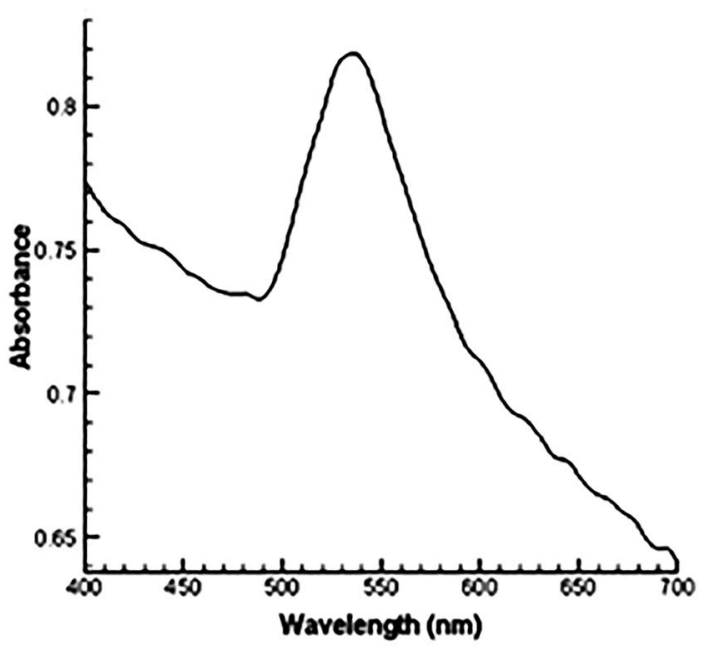

(d)

Fig. 3 The UV-vis spectrometry analysis for the fungi a Paecilomyces variotii, b Peceaiomyces sp. c Sclerotinia sclerotium, d Verticillium dahliae

Table 3 The wavelength of the maximum absorption peak for the examined fungi

\begin{tabular}{lll}
\hline Row & Fungus & $\begin{array}{l}\text { Wave- } \\
\text { length } \\
(\mathrm{nm})\end{array}$ \\
\hline 1 & Fusarium oxysporum & 530 \\
2 & Sclerotinia sclerotium & 526 \\
3 & Aspergillus flavus & 534 \\
4 & Paecilomyces variotii & 539 \\
5 & Colletotrichum gloeosporioides & 525 \\
6 & Rhizoctonia solani & 520 \\
7 & Verticillium dahliae & 534 \\
8 & Peceaiomyces sp. & 537 \\
\hline
\end{tabular}

of the fungus used in their study. As mentioned before, the difference between the species can also affect the size of the nanoparticles.

\section{Conclusion}

In this study, the capability of 12 fungi in the synthesis of gold nanoparticles was evaluated based on the color change observational method and UV-vis spectrometry. These evaluations of the fungi showed that out of 12 fungi scrutinized, 8 can synthesize gold nanoparticles. In the next stage, the fungi Fusarium oxysporium, Aspergillus flavus, Rhizoctonia solani, and Verticillium dahlia were selected for further investigation via transient electron 
Fig. 4 The TEM micrographs for gold nanoparticles synthesized by the fungi a Verticillium dahliae, b Fusarium oxysporum, c Aspergillus flavus, d Rhizoctonia solani

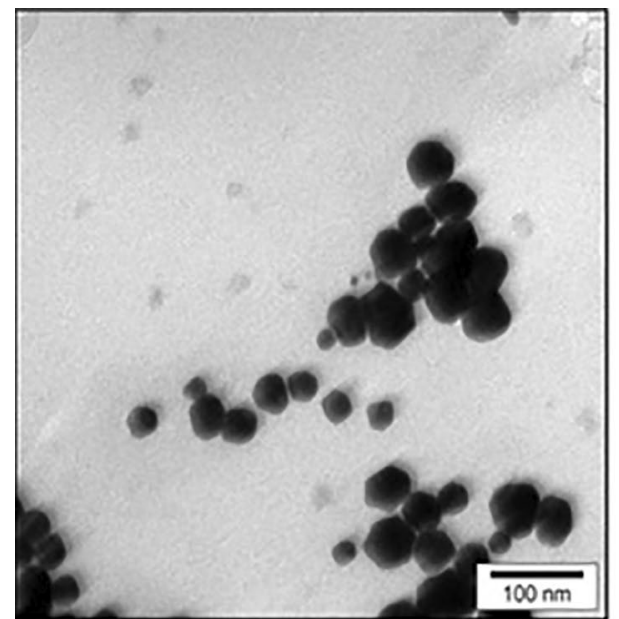

(a)

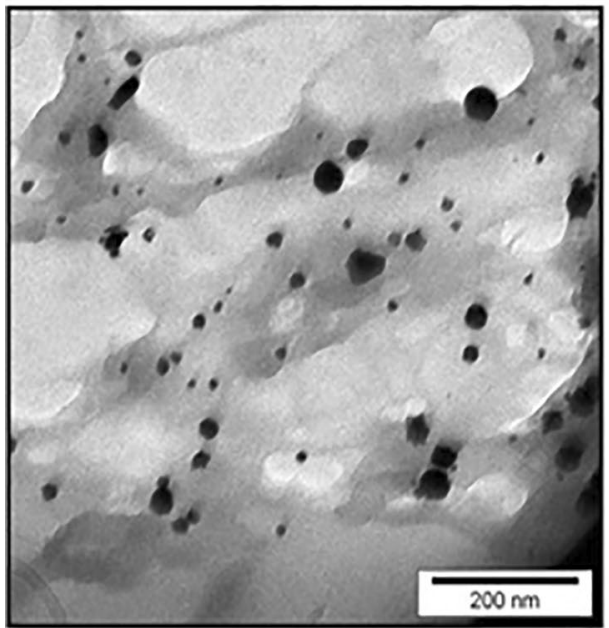

(c)

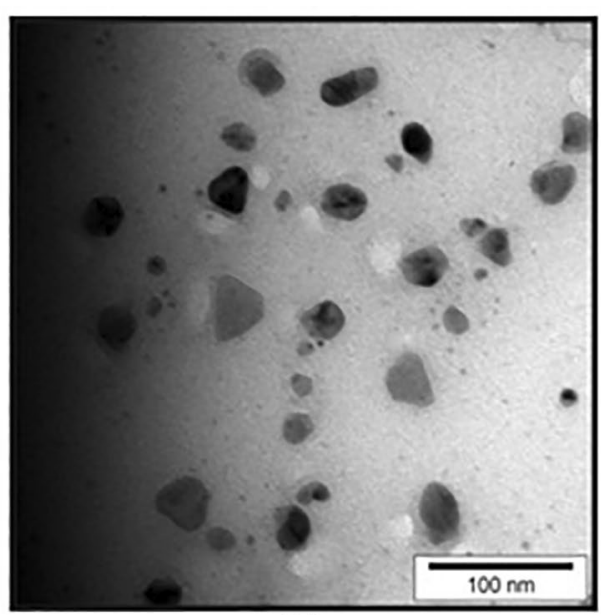

(b)

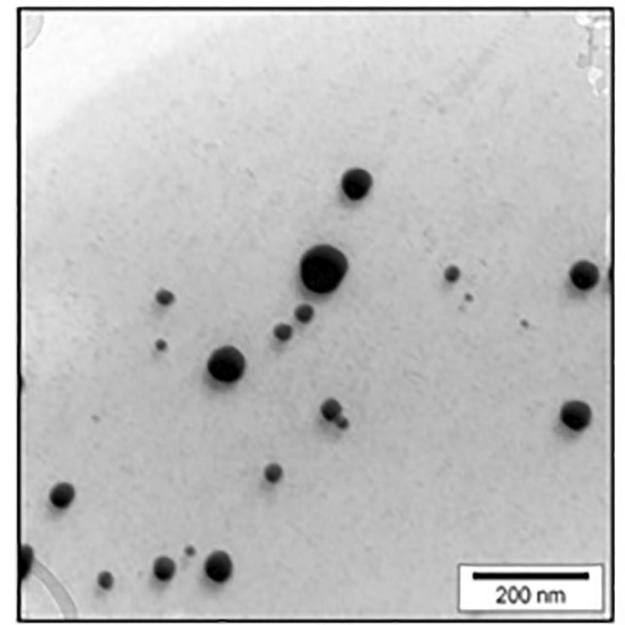

(d) microscope (TEM) and X-ray diffraction (XRD) analyses. The main results of this research can be summarized as follows:

1 In the UV-vis spectrometry, the maximum and minimum amounts of absorption belonged to the fungus Rhizoctonia solani and Peceaiomyces sp., respectively. According to the TEM micrographs, the average size of the nanoparticles for the fungi Aspergillus flavus and Rhizoctonia solani was about $30 \mathrm{~nm}$. In addition, Fusarium oxysporum and Verticillium dahlia synthe- sized the nanoparticles with an average size of 20 and $40 \mathrm{~nm}$, respectively. The synthesized nanoparticles for the examined fungi were intercellular with spherical, triangular and polygonal shapes. The maximum number of the synthesized nanoparticles belonged to the fungus Rhizoctonia solani.

2 Based on the XRD analysis, the synthesized particles are gold nanocrystals.

3 The species of the fungus affects the size of the synthesized nanoparticles. 


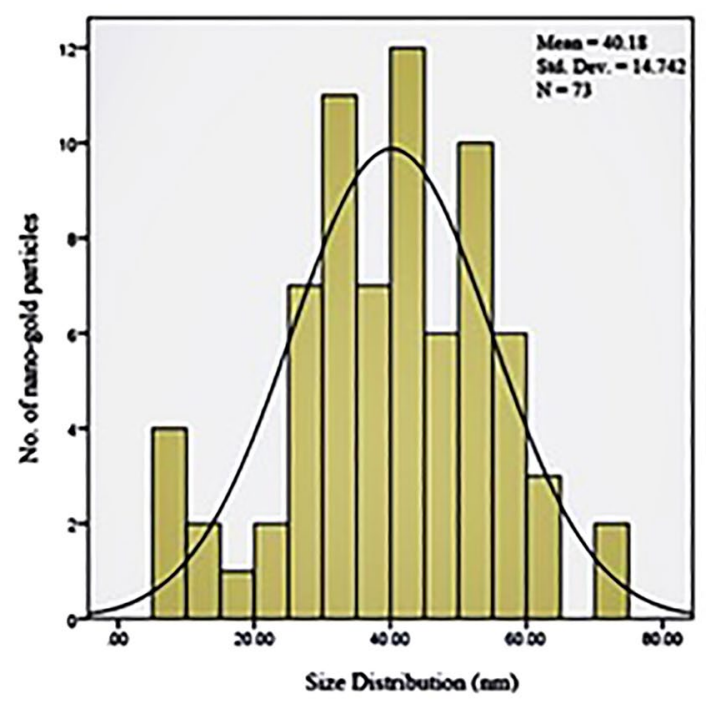

(a)

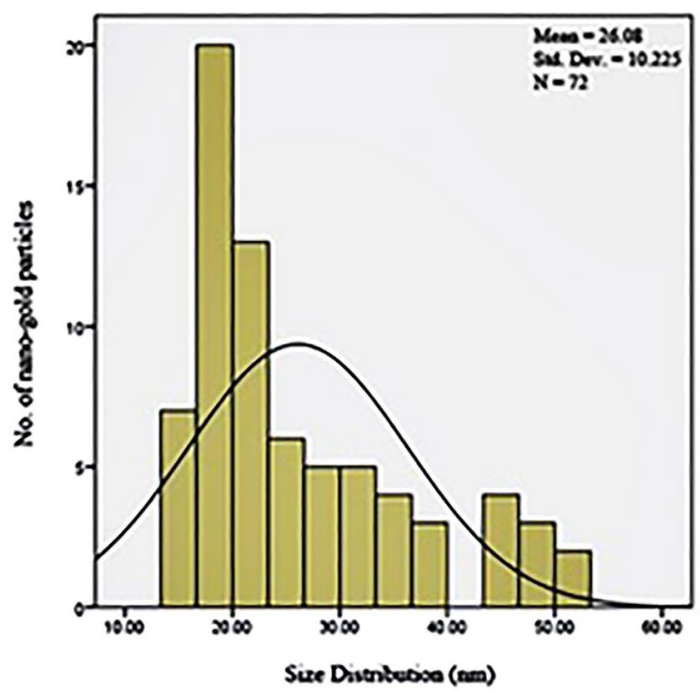

(c)

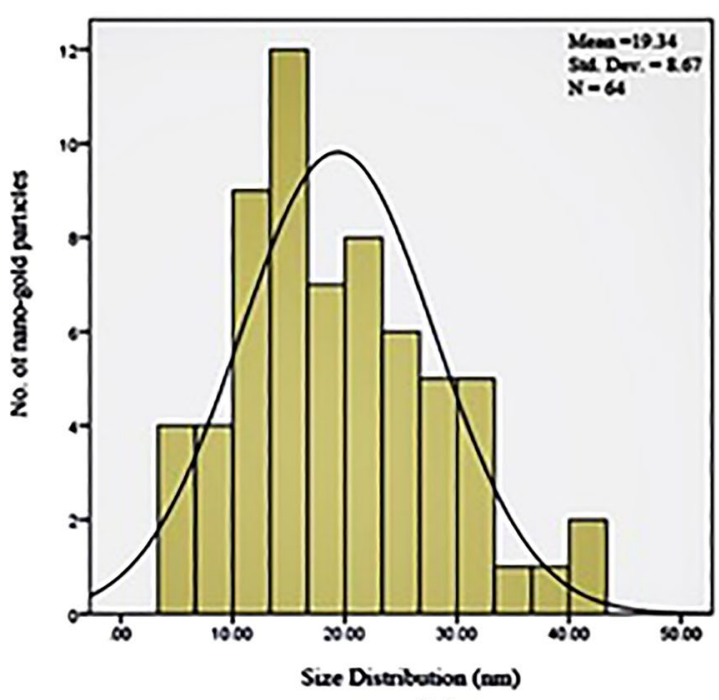

(b)

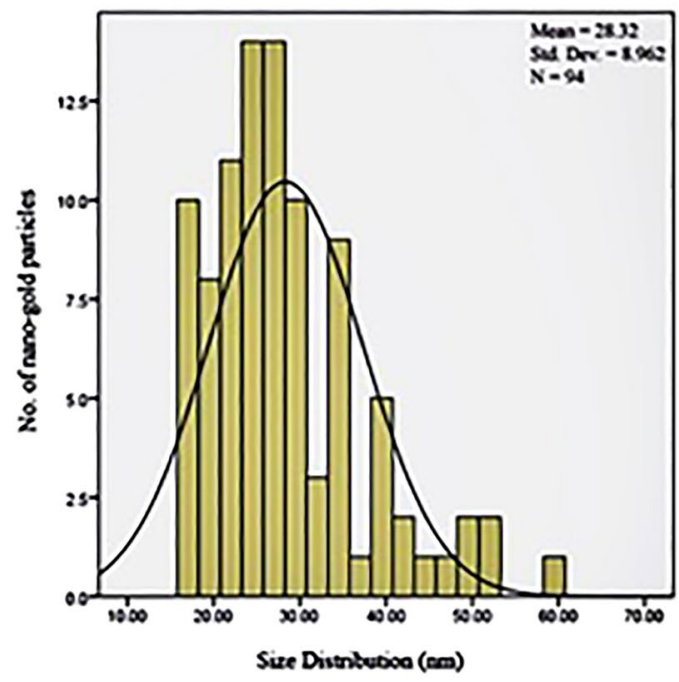

(d)

Fig. 5 Histogram of the size distribution of gold nanoparticles synthesized by the fungi a Verticillium dahliae, b Fusarium oxysporum, c Aspergillus flavus, d Rhizoctonia solani 
Fig. 6 XRD pattern for gold nanoparticles synthesized by the fungi a Aspergillus flavus, b Fusarium oxysporum

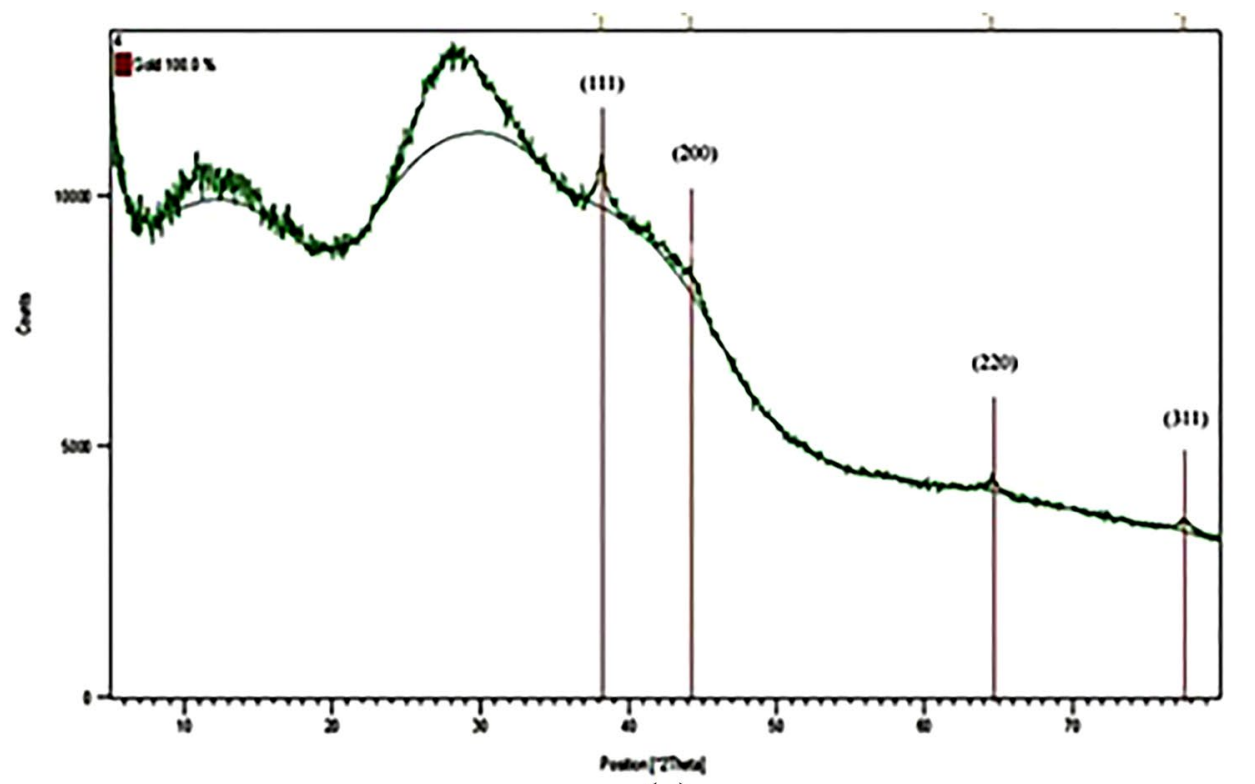

(a)

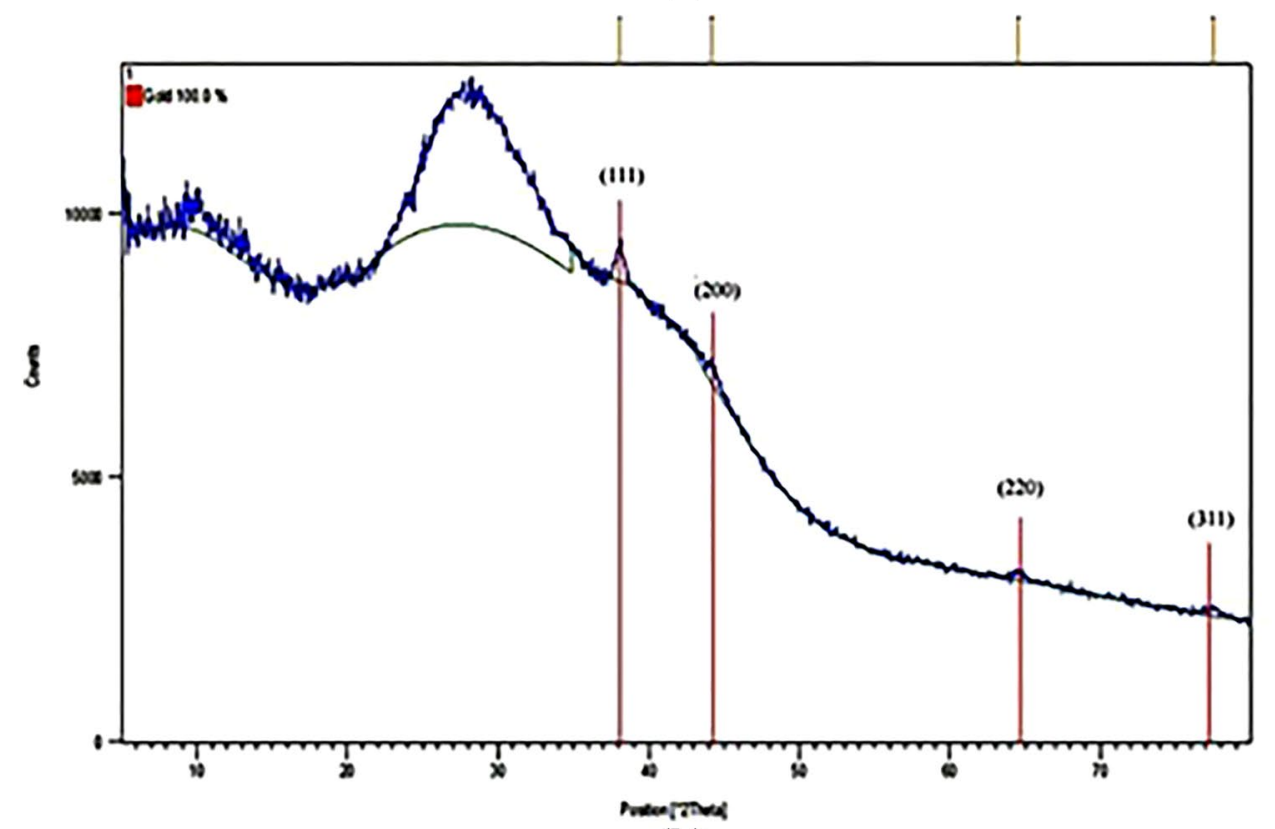

(b) 
Fig. 7 XRD pattern for the gold nanoparticles synthesized by the fungi a Rhizoctonia solani, b Verticillium dahliae

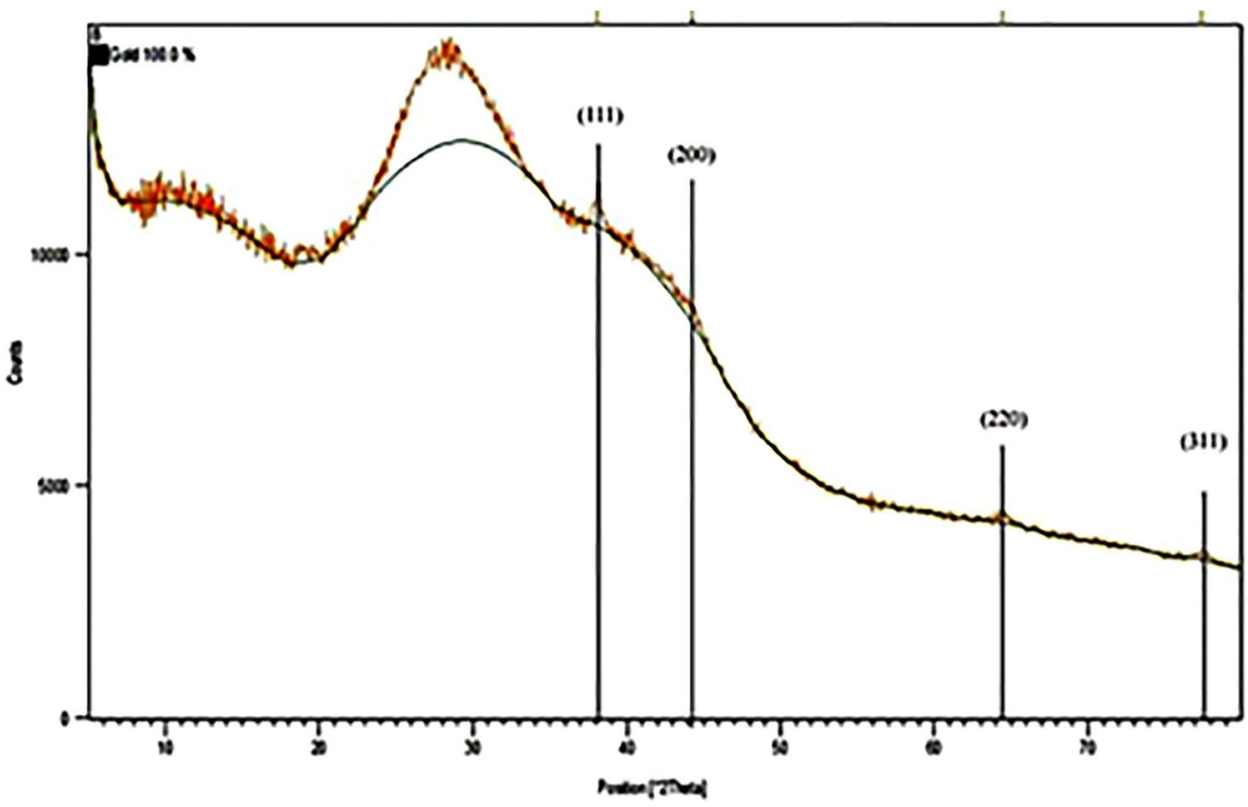

(a)

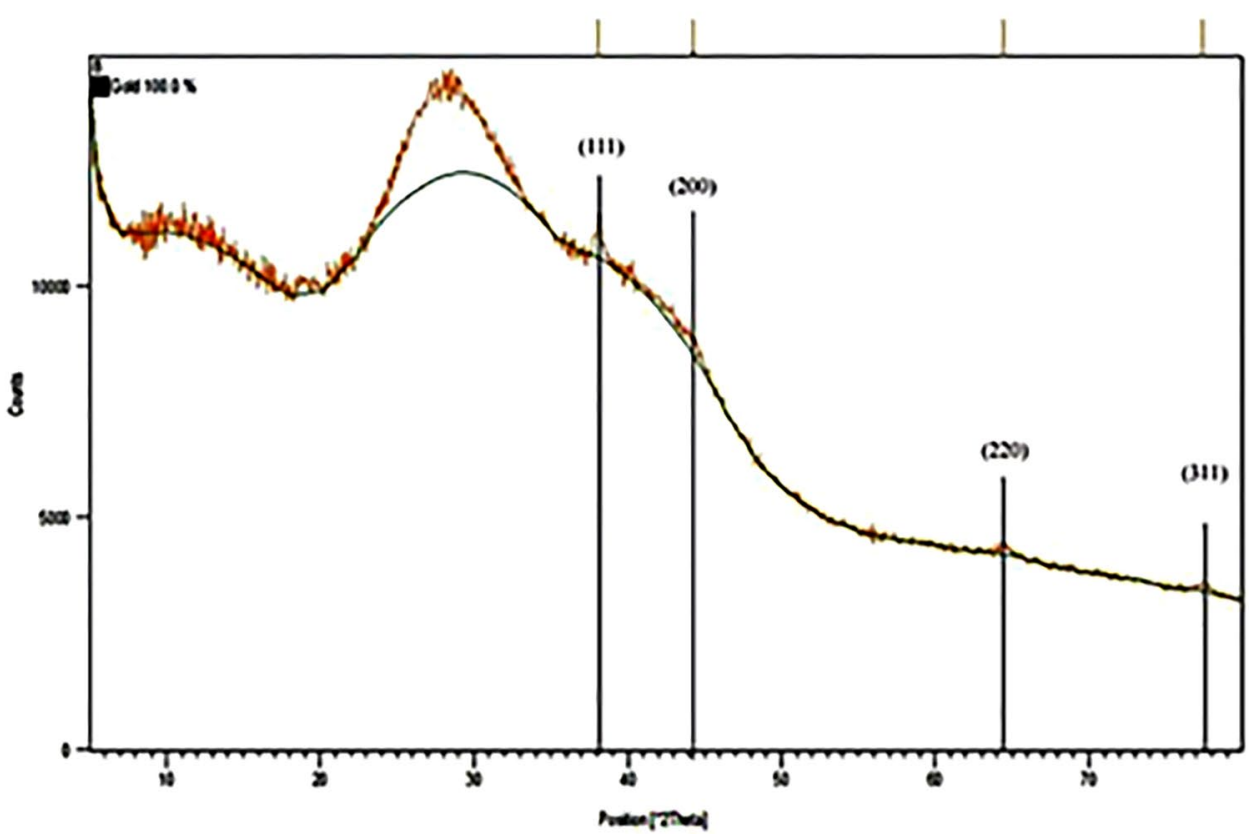

(b)
Acknowledgements This research was financially supported by Iran National Science Foundation Grant No. 91003706.

\section{Compliance with ethical standards}

Conflict of interest The authors declare that there is no conflict of interests regarding the publication of this manuscript.

\section{References}

1. Mohanpuria P, Rana NK, Yadav SK (2008) Biosynthesis of nanoparticles: technological concepts and future applications. J Nanopart Res 10:507-517

2. Mukherjee P, Ahmad A, Mandal D, Senapati S, Sainkar SR, Khan MI, Ramani R, Parischa R, Ajayakumar PV, Alam M, Sastry M (2001) Bioreduction of AuCl4-ions by the fungus, Verticillium sp. and surface trapping of the gold nanoparticles formed. Angew Chem Int Edition 40:3585-3588

3. Mukherjee P, Senapati S, Mandal D, Ahmad A, Khan Ml, Kumar R, Sastry M (2002) Extracellular synthesis of gold nanoparticles by the fungus Fusarium oxysporum. ChemBioChem 3:461-463 
4. Shankar SS, Ahmad A, Pasricha R, Sastry M (2003) Bioreduction of chloroaurate ions by geranium leaves and its endophytic fungus yields gold nanoparticles of different shapes. J Mater Chem 13:1822-1826

5. Ahmad A, Senapati S, Khan MI, Kumar R, Sastry M (2005) Extra-/intracellular biosynthesis of gold nanoparticles by an alkalotolerant fungus, Trichothecium sp. J Biomed Nanotechnol 1:47-53

6. Bhambure R, Bule M, Shaligram N, Kamat M, Singhal R (2009) Extracellular biosynthesis of gold nanoparticles using Aspergillus niger-its characterization and stability. Chem Eng Technol 32:1036-1041

7. Philip D (2009) Biosynthesis of Au, Ag and Au-Ag nanoparticles using edible mushroom extract. Spectrochim Acta A Mol Biomol Spectrosc 73:374-381

8. Binupriya AR, Sathishkumar M, Yun S-I (2010) Biocrystallization of silver and gold ions by inactive cell filtrate of Rhizopus stolonifer. Colloids Surf B Biointerfaces 79:531-534

9. Soni N, Prakash S (2012) Synthesis of gold nanoparticles by the fungus Aspergillus niger and its efficacy against mosquito larvae. Rep Parasitol 2:1-7

10. Sarkar J, Ray S, Chattopadhyay D, Laskar A, Acharya K (2012) Mycogenesis of gold nanoparticles using a phytopathogen Alternaria alternata. Bioprocess Biosyst Eng 35:637-643

11. Thakker JN, Dalwadi P, Dhandhukia PC (2013) Biosynthesis of gold nanoparticles using Fusarium oxysporum f. sp. cubense JT1, a plant pathogenic fungus. Int Sch Res Notices 2013:1-5
12. Gopinath K, Arumugam A (2014) Extracellular mycosynthesis of gold nanoparticles using Fusarium solani. Appl Nanosci 4:657-462

13. Joshi CG, Danagoudar A, Poyya J, Kudva AK, Dhananjaya BL (2017) Biogenic synthesis of gold nanoparticles by marine endophytic fungus-Cladosporium cladosporioides isolated from seaweed and evaluation of their antioxidant and antimicrobial properties. Process Biochem 63:137-144

14. Patil MP, Kim GD (2018) Marine microorganisms for synthesis of metallic nanoparticles and their biomedical applications. Colloids Surf B Biointerfaces 172:487-495

15. Pourali P, Yahyaei B, Afsharnezhad S (2018) Bio-synthesis of gold nanoparticles by Fusarium Oxysporum and assessment of their conjugation possibility with two types of $\beta$-Lactam antibiotics without any additional linkers. Microbiology 87:229-237

16. Sreedharan SM, Gupta S, Saxena AK, Singh R (2019) Macrophomina phaseolina: microbased biorefinery for gold nanoparticle production. Ann Microbiol 69:435-445

17. Mulvaney $P$ (1996) Surface plasmon spectroscopy of nanosized metal particles. Langmuir 12:788-800

Publisher's Note Springer Nature remains neutral with regard to jurisdictional claims in published maps and institutional affiliations. 